\title{
Domestic Refrigerators Temperature Prediction Strategy for the Evaluation of the Expected Power Consumption
}

Lakshmanan, Venkatachalam; Marinelli, Mattia; Kosek, Anna Magdalena; Sossan, Fabrizio; Nørgård, Per Bromand

\section{Published in:}

Proceedings of the 2013 4th IEEE PES Innovative Smart Grid Technologies Europe

Publication date:

2013

Link back to DTU Orbit

Citation $(A P A)$ :

Lakshmanan, V., Marinelli, M., Kosek, A. M., Sossan, F., \& Nørgård, P. B. (2013). Domestic Refrigerators Temperature Prediction Strategy for the Evaluation of the Expected Power Consumption. In Proceedings of the 2013 4th IEEE PES Innovative Smart Grid Technologies Europe IEEE.

\section{General rights}

Copyright and moral rights for the publications made accessible in the public portal are retained by the authors and/or other copyright owners and it is a condition of accessing publications that users recognise and abide by the legal requirements associated with these rights.

- Users may download and print one copy of any publication from the public portal for the purpose of private study or research.

- You may not further distribute the material or use it for any profit-making activity or commercial gain

- You may freely distribute the URL identifying the publication in the public portal 


\title{
Domestic Refrigerators Temperature Prediction Strategy for the Evaluation of the Expected Power Consumption
}

\author{
Venkatachalam Lakshmanan, Mattia Marinelli, Anna Magdalena Kosek, Fabrizio Sossan, Per Nørgård \\ Center for Electric Power and Energy \\ Technical University of Denmark, \\ Roskilde, Denmark \\ \{vela; matm; amko; faso; pern\}@elektro.dtu.dk
}

\begin{abstract}
This paper discusses and presents a simple temperature prediction strategy for the domestic refrigerator. The main idea is to predict the duration it takes to the cold chamber temperature to reach the thresholds according to the state of the compressor and to the last temperature measurements. The experiments are conducted at SYSLAB facility at DTU Risø Campus having a set of refrigerators working at different set point temperatures, with different ambient temperatures and under different thermal load conditions. The prediction strategy is tested using a set of different refrigerators in order to validate the performances. The challenges to calculate the time with less error pronouncement in temperature, regulating power supply and its duration are also discussed.
\end{abstract}

Index Terms-Regulating power, Energy Storage, Grid Integration, Demand response.

\section{INTRODUCTION}

The rise in energy demand, the threat of depleting fossil fuels and their impairment to the environment is pushing the usage of more renewable energy sources with increased small distributed production units rather than large localized traditional power plants. In Denmark solar and wind generation accounted to $21 \%$ in 2010 and ambitious national targets suggest $50 \%$ consumption to be supplied from wind in 2020 [1]. Increase of an uncontrollable and variable power production disturbs balance between supply and demand and a shift to production driven consumption is inevitable. Smart Grid technology is expected to provide benefits by reducing distribution and transmission losses, and help to optimize the use of existing power grid infrastructure [2].

One of the challenges for the reliable and economical operation of a power system with a high penetration of renewable energy sources with high fluctuations (solar, wind) is the availability of energy resources which can participate in balancing supply and demand on a short-term basis. Demand response paradigm suggests that such resources can be found on the demand side rather only in the supply side. The

This work is part of INCAP project (INducing Consumer Adoption of automated reaction technology for dynamic Power pricing tariffs), funded by The Danish Council for Strategic Research, running from 2012 to 2016. increase of not controllable variable power production and the displacement of conventional generation require power system services, such as regulating power provision, to be reallocated. A possible source of regulating power is offered by so called Demand Side Resources (DSRs), which are electric loads whose power consumption can be deferred without compromising the quality of the services they are providing to the users. Demand response paradigm is based on the idea that contribute in terms of electric power of the single unit is modest but the aggregated response of a very large number of units might be of relevant size for the power system [3]. In Nordic countries in 2010 buildings energy consumption was $34 \%$ of total consumption, where appliances and miscellaneous equipment accounted to $24 \%$ of energy consumption in the building sector [4]. Among appliances found in a household, refrigerators, washing machines, dryers and dishwashers are considered to be suitable for load shifting [5]. Loads with thermal capacity and continuous operation are even more suitable for shifting. Using, for example, a thermal energy storage capacity in a refrigerator, consumption can be shifted away from peak hours, while managing a stable temperature within set range.

Availability of a refrigerator in most of households, ability to shift its consumption due to presence of thermal storage and continuity of operation, with thermostat cycles, makes a refrigerator a suitable unit for a large field experiment controlling appliances in private premises. The main challenge in such a context is to predict if and for how long a refrigerator is available to defer its power consumption. In order to have a realistic estimate of the fridge power consumption flexibility, a thermal model for predicting the future temperature of the cold vane can be used.

The aim of this paper is to present a simple thermal prediction strategy of a refrigerator that can capture its main thermal dynamics. The strategy will be validated on real domestic refrigerator units which work at different indoor and ambient temperatures and under different thermal load conditions. Particular emphasis will be put on creating an adaptive model which can estimate and update its parameters 
on-line. The rest of this paper is organized as follows: in Section II the problem outline and prediction strategy are introduced, and the thermal model of the fridge is briefly presented. Section III illustrates how the work will be carried out and preliminary experimental data which were used for fitting the model. Section IV discusses about the work plan in detail and the conclusions and future work are reported in section $\mathrm{V}$.

\section{PREDICTION STRATEGY}

\section{A. Problem outline and prediction strategy description}

The domestic refrigerator's operation during most of the time of a day is very much defined by the operation of the thermostat. The major disturbances are introduced only during certain time of the day while the food is removed from and fresh food is loaded into the fridge. During rest of the day, the internal temperature of the fridge is not affected by user behavior and it can be predicted using a simple strategy tuned with historical measurements. This allows predicting the duration for which the regulatory power can be supplied or consumed.

The quantity of the available regulating power is the fridge power consumption at any time. Regulating power supply duration is the duration for which the fridge operation can be interrupted without violating upper and lower threshold of internal temperature of the fridge. The regulating power supply duration can be estimated by predicting the time that it takes for the temperature to reach upper or lower threshold of the respective thermal cycle from the present temperature inside the cold chamber. Thermal cycles examples are shown in Figure 2 with cooling cycle between time $t_{1}$ and $t_{2}$, and heating cycle between time $t_{2}$ and $t_{3}$. Because of the thermostatic cycles, the thermal behavior of the fridge follows the earlier respective heating or cooling cycle with minor or no variations. In the work presented in this paper, predictions for the present heating and cooling cycle are based on the previous respective cycles.

To predict the duration of present cycle, the previous respective cycle's temperature curve can be traced till the threshold temperatures of the cycle and applied on the present cycle. For example, in the illustration shown in Figure 2, to predict the cooling cycle between the time period $t_{3}$ and $t_{5}$, previous cooling cycle from time period $t_{1}$ to $t_{2}$ can be used. Similarly, to predict the heating cycle between the time period $t_{5}$ and $t_{6}$, previous heating cycle from time period $t_{2}$ to $t_{3}$ can be used. The shape of the temperature curve is characterized by its slope at different segments. One of the ways to trace the temperature curves of these cycles is by considering them a piecewise linear. The slopes can be derived for each segment of a curve by considering the segment as a linear with one slope. These slopes of different segments of the previous cycle can be used to construct the temperature curve of the cycle under prediction.

The block diagram in Figure 1 shows the prediction strategy with the expected input and output quantities with temperature prediction strategy that is discussed in this paper.

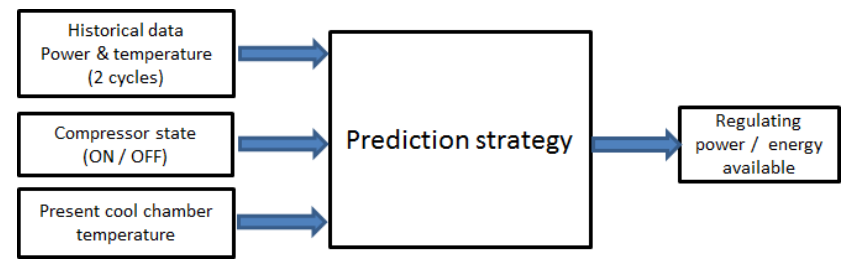

Figure 1. Block diagram of fridge model showing input and output.

\section{B. Prediction error}

The temperature prediction error has a direct impact on the prediction of regulating power supply duration. The prediction error in duration is calculated as shown in Figure 2. In the cooling cycle, the prediction error is the ratio of the difference in time between the actual temperature and the predicted temperature reaching lower threshold temperature and the actual cooling duration. Similarly, in the heating cycle the prediction error is the ratio of the difference in time between the actual temperature and the predicted temperature reaching higher threshold temperature and the actual heating duration. The errors in prediction are defined as follows:

$$
\text { Prediction error }=\Delta t_{c, h} / t_{c, h}
$$

Where $\Delta t_{c, h}$ is the difference between actual time and the predicted time for the temperature to reach upper or lower threshold temperature and $t_{c, h}$ is actual heating or cooling cycle duration. The positive error represents the under estimation and vice versa.

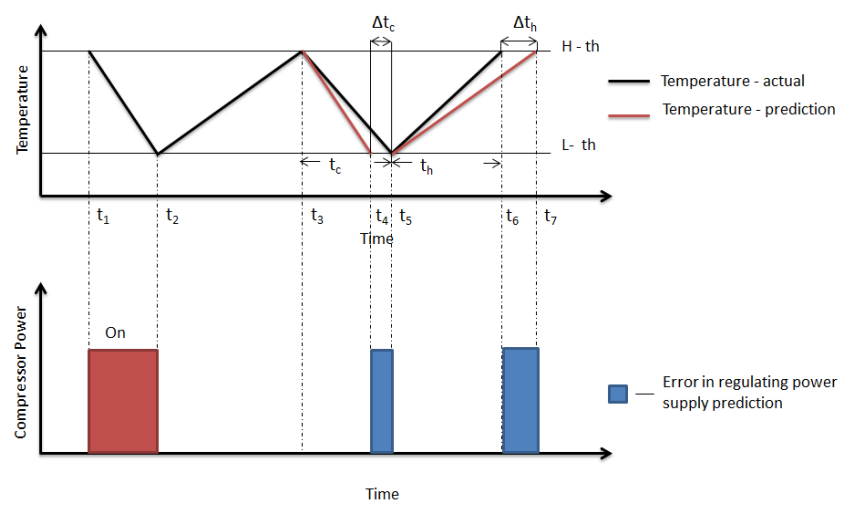

Figure 2. Prediction error calculation in cycle duration.

\section{EXPERIMENTAL SETUP}

Preliminary measurements of two consecutive thermostatic cycle of one refrigerator unit are shown in Figure 3. Upper plot of Figure 3 shows the cold chamber temperature while lower plot shows the refrigerator power consumption. The power consumption shows an initial spike due to the inrush current of the induction motor which drives the compressor. Electric power consumption is afterwards decreasing along with the rate of the fridge indoor temperature. Time series of the electric power consumption and fridge indoor temperature is used for fitting on-line the refrigerator prediction strategy. 

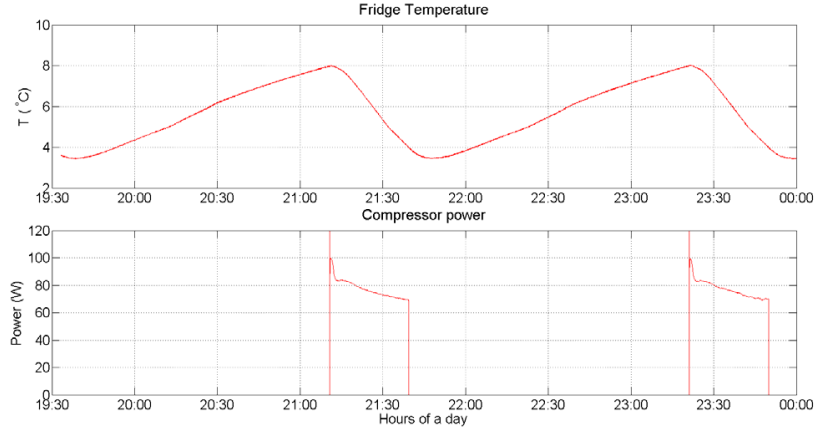

Figure 3. Fridge temperature profile and compressor power profile for two cycles.

In this paper the proposed thermal model will be fitted using two refrigerator units at different working conditions (cool chamber temperature set point, ambient temperature, food thermal mass) and its performances, accuracy and reliability in estimating the indoor temperature and refrigerator power consumption is assessed.

\section{A. Measurement and data collection}

The aim of the presented experiments is to collect different set of temperature data to train the model and test the selftunability (to adopt the changes in variations in the cycle) of the model for the temperature prediction in order to calculate the duration of regulating power supply. The prediction error in terms of heating and cooling duration can be calculated from the actual cycle time and the predicted time. The block diagram of the experimental setup is shown in the Figure 4.

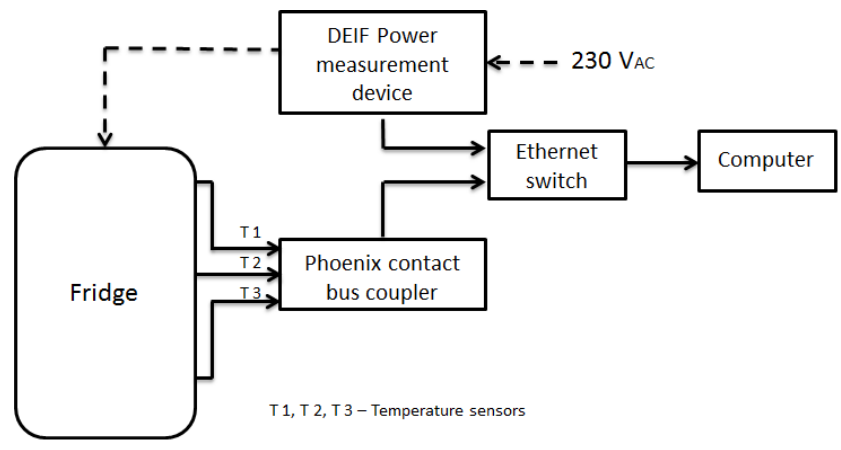

Figure 4. Experimental setup and sensor position.

The power was measured with Multi-instrument, MIC-2 from DEIF A/S with accuracy Class 0.2. The temperature was measured with TEL NTC 10 thermistors from Produal A/S with temperature range from -30 to $80{ }^{\circ} \mathrm{C}$ and accuracy $\pm 0.2^{\circ} \mathrm{C}$ (at $25^{\circ} \mathrm{C}$ ) connected to Phoenix bus coupler which reads the resistance of the thermistors. The temperature data and the power consumption along with a common time stamp were logged in a CSV file.

\section{EXPERIMENTS}

\section{A. Sensor characterization and placement}

The temperature measurement was done with three sensors. To ensure there is no considerable difference in their readings, they were placed together in single place inside of the cold chamber and the measurement of the same temperature was taken from different sensors. The measurements from the sensors and the difference in their readings are shown in Figure 5. Two of the sensors were measuring the temperature with difference of $\pm 0.2{ }^{\circ} \mathrm{C}$ and the third one differs with the others with $\pm 0.5^{\circ} \mathrm{C}$.
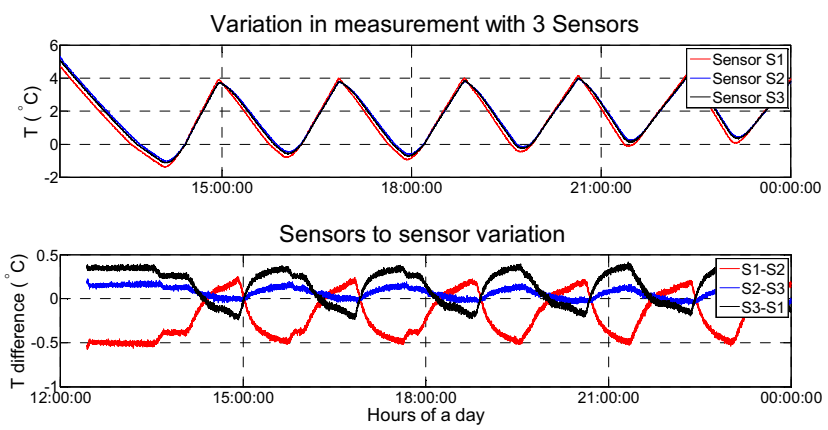

Figure 5. Temperature measurement variation in the sensors.

\section{B. Experiment plan}

In order to properly place the sensor and to minimize the prediction error, large sets of experimental data were analyzed. The fridge temperature was measured at three different places inside the cold chamber. Based on measurements presented in Figure 5, the final placement of the sensors for the experiment was determined. One measurement was placed close to the cooling element (the evaporator), the second one was as much as far away from the cooling element and the third one was in between the former two sensors. The sensor which gives more variation was placed in the middle of the cold chamber whereas the other two sensors were placed at top and bottom of the cold chamber of the fridge during the experiment.

Two fridges with different thermal characteristics were selected. One fridge of volumetric capacity of 75 liters with separate doors for fridge and freezer compartments, in which the temperature is controlled by the thermostat mounted in the freezer and the fridge compartment is separated from the freezer with high thermal conductive layer compared to the fridge wall insulation layer. The fridge compartment is above the freezer which is the cooling element and the coldness flows from the bottom to the top. This unit is called Fridge-1. The second fridge of volumetric capacity of 50 liters with a single door, evaporator is mounted inside the fridge cold chamber. The evaporator is the cooling element and the freezer. Here the coldness flows from the top to the bottom. This unit is called Fridge- 2 in the experiment. The Figure 6 shows the construction and direction of coldness flow in Fridge-1 and Fridge-2.

The thermostat settings in refrigerators from different manufacturers are not following common set points indications. Some fridges have only qualitative indications of coolness only allowing setting low, medium and high. Some of the fridges have numeric coolness indicators, for example numbers 1 to 5 , where 1 is the lowest and 5 is the highest temperature setting. It was decided to conduct the experiment at three different levels of thermostat setting and with three 
different levels of thermal mass, in order to examine fridge flexibility with different settings and loads.

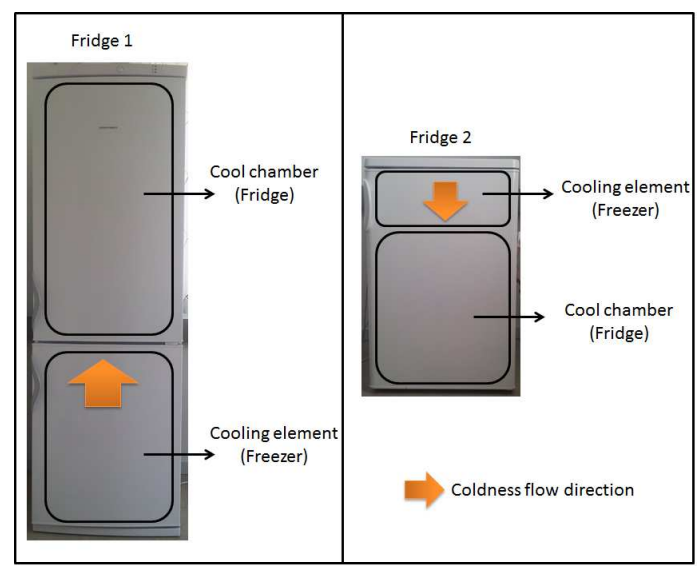

Figure 6. Construction and heat flow direction in Fridge-1 and Fridge -2.

\section{Inference from the temperature cycle}

The temperature cycles of cold chamber of the two fridges during a full day operation are shown Figure 7 and Figure 8. The temperature variations at different zones of the fridge are different.
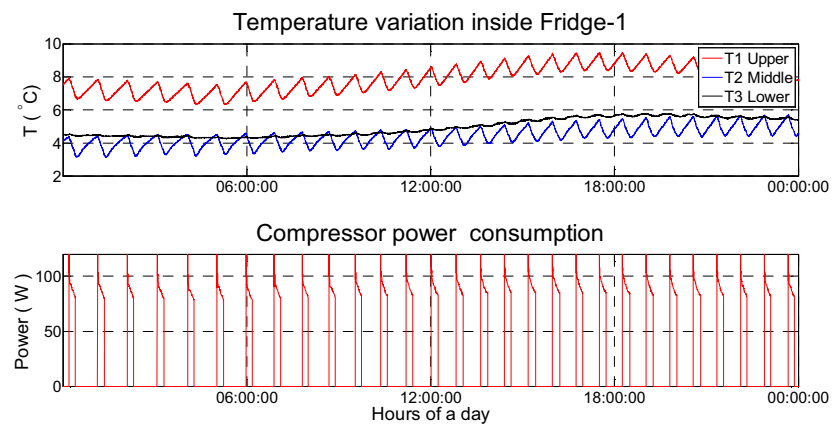

Figure 7. Temperature variation in Fridge-1.

The variations in the temperature are more pronounced in the middle than the one at the top or bottom. Figure 7 shows the measurements taken in the fridge in which the cooling element is at the bottom and in the Figure 8, shows the measurements in a fridge in which the cooling element is at the top. Also in the fridge driven by the freezers (Figure 7), the upper and lower threshold varies in wide range with the change in ambient temperature.
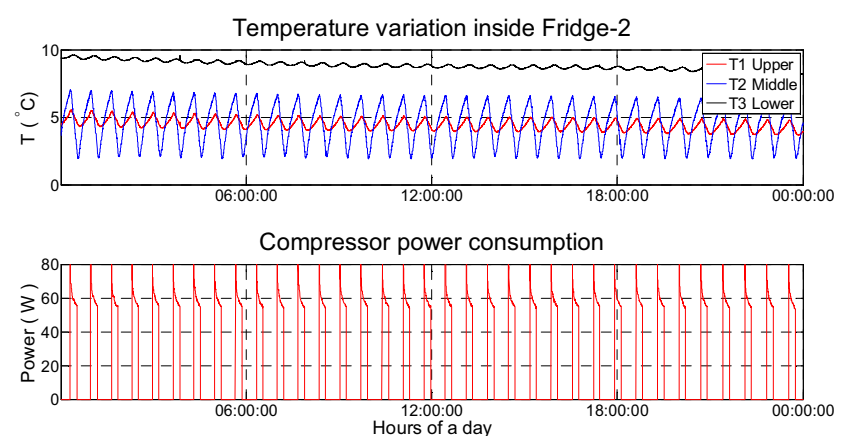

Figure 8.Temperature variation in Fridge-2.

\section{Temperature prediction for Fridge-1}

The temperature curves of cooling cycle and the heating cycle follows different trends. They are considered as two different systems. Predictions for the present heating and cooling cycle are based on the previous respective cycles. The measurement from sensor mounted at the middle of the cold chamber was used for the temperature prediction as the temperature cycles are more visible in the measurement. The temperature curve of a cycle is traced by its slopes at different segments and the slopes were used to construct the curve under prediction. The Figure 9 shows the closer view of actual temperature, predicted temperature for two cycles of cooling and heating.

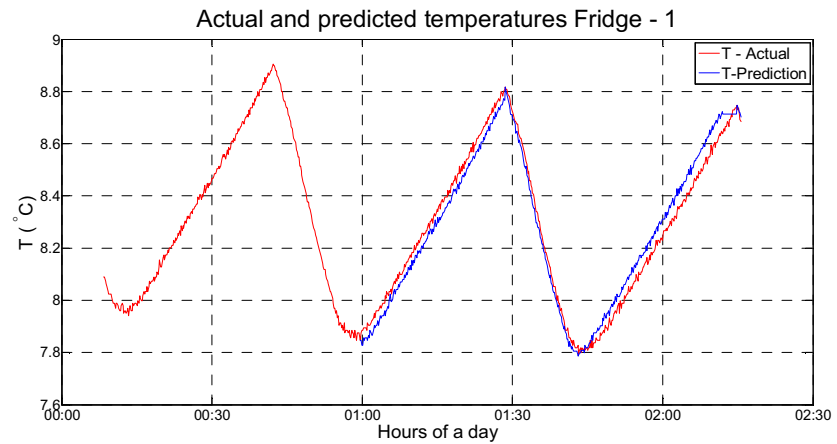

Figure 9. Temperature prediction for two cycles in Fridge-1.

The Figure 10 shows the actual temperature, predicted temperature and the difference between them for a whole day period.
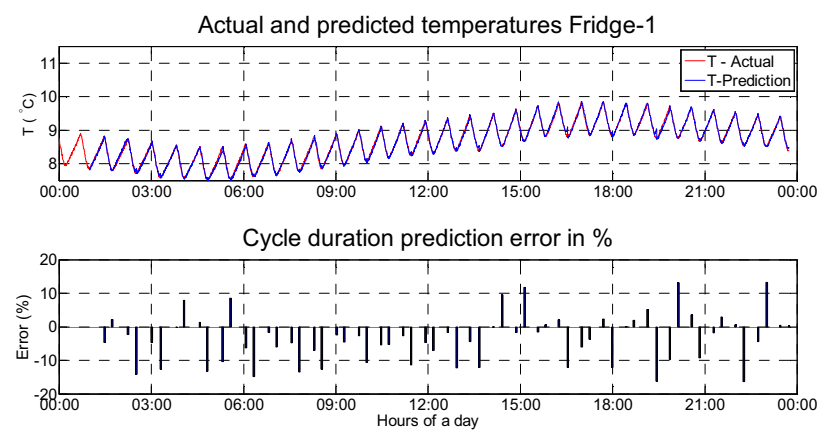

Figure 10. Temperature prediction and prediction error in Fridge-1 for the full day.

The mean errors and the standard deviations for the prediction for a full day are given in the TABLE 1 .

TABLE 1. PREDICTION ERROR FOR FRIDGE-1

\begin{tabular}{|l|c|c|c|}
\hline & Mean error & Standard deviation & Maximum error \\
\hline Cooling & $-7.4 \%$ & $14.2 \%$ & $16.1 \%$ \\
\hline Heating & $-2.3 \%$ & $3.6 \%$ & $3.2 \%$ \\
\hline
\end{tabular}

\section{E. Temperature prediction for Fridge-2}

The ability of the model to predict the temperature was tested with the Fridge- 2 in normal operation and with user interaction. The fridge was partially loaded $(60 \%$ by the volumetric capacity, i.e. $18 \mathrm{~L}$ of water) with water bottles and 
the thermostat was set at medium level. After the regular cooling and heating cycles were achieved, the disturbances were introduced. The disturbances were:

- Random door openings for the duration of 30 seconds and 60 seconds.

- Removal of thermal mass (12.5\% of present mass)

- Addition of thermal mass (5\% of present mass)

- Addition of thermal mass (13\% of present mass)

The prediction for a whole day with normal operation without any user interaction is shown in the Figure 11. The prediction error means and standard deviations are given in the Table 2 .

TABLE 2. PREDICTION ERROR FOR FRIDGE-2

\begin{tabular}{|l|c|c|c|}
\hline & Mean error & Standard deviation & Maximum error \\
\hline Cooling & $-1.0 \%$ & $4.8 \%$ & $9.5 \%$ \\
\hline Heating & $0.5 \%$ & $5.5 \%$ & $10.4 \%$ \\
\hline
\end{tabular}

The first three disturbances mentioned above were introduced between 08:00 and 12:00 hours of the day and have no considerable impact on the prediction error which is shown in Figure 11.
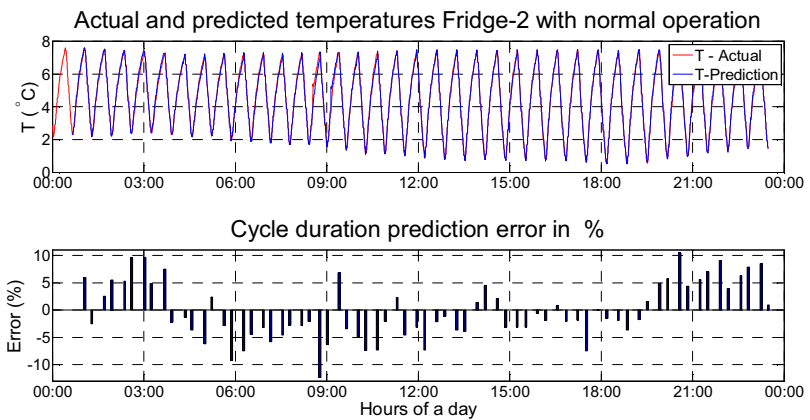

Figure 11. Temperature prediction and prediction error in Fridge-2 with normal operation for a day.

Addition of $13 \%$ thermal mass, causes a huge drift in the lower and upper thresholds of the thermal cycles, the prediction error was above $40 \%$ in the first two cycles after the change in thermal mass as shown in Figure 12. The actual temperature, the predicted one and their difference are shown in the Figure 12. The prediction error means and standard deviations are given in the Table 3.

TABLE 3. PREDICTION ERROR FOR FRIDGE-2 WITH USER INTERACTION

\begin{tabular}{|l|c|c|c|}
\hline & Mean error & Standard deviation & Maximum error \\
\hline Cooling & $-0.5 \%$ & $10.8 \%$ & $9.5 \%$ \\
\hline Heating & $-0.3 \%$ & $6.1 \%$ & $47.5 \%$ \\
\hline
\end{tabular}

The prediction errors are less pronounced for the fridges where the thermostat is mounted in the cold chamber where the measurement is taken for the prediction as the variation in the upper and lower threshold temperature are less.
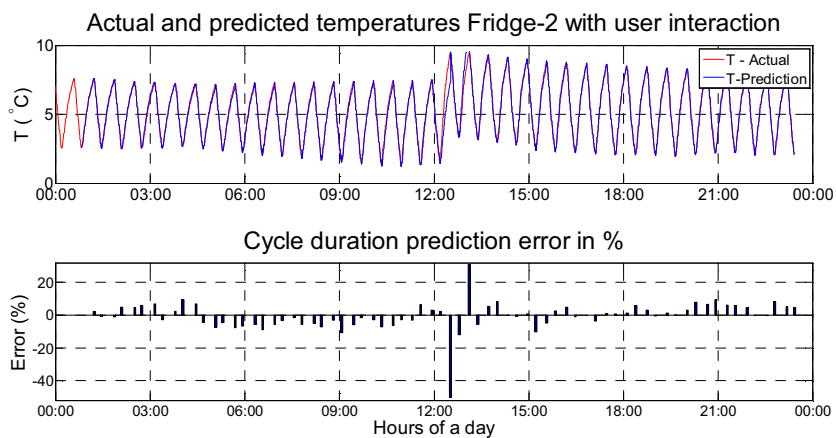

Figure 12. Temperature prediction and prediction error in Fridge-2 with user interaction.

\section{CONCLUSIONS AND FUTURE WORK}

The paper main idea is to predict the duration that it takes for the cold chamber temperature to reach the lower or the upper threshold temperatures, according to the state of the compressor and the series of temperature measurements from the last cooling or heating cycle. The aim is to be able to predict the temperature behavior of large set of fridges, and thus evaluate the expected power consumption. The presented fridge model was fitted with experimental data from a test fridge. The proposed prediction strategy for power consumption estimation was verified using two different fridges and the results showed a standard deviation in the prediction error of $14.2 \%$ and $4.8 \%$ for the cooling cycle. The heating cycle errors are respectively $3.6 \%$ and $5.5 \%$.

The prediction strategy used here is simple and needs little computing power and only two measurements: temperature and compressor state, but it is usable for predicting the power consumption of a fridge of unknown type, size and load, fitting its prediction to previous heating and cooling cycles. From the results obtained, it seems that the method is more suitable for the fridges where the thermostat is mounted in the cold chamber, such as Fridge- 2 . The temperature sensor cables cause thermal leak in the system which introduces minor disturbances in to system. Usage of wireless temperature sensors can solve this problem. Further field implementations are aimed at evaluating aggregated behavior of a larger set of fridges in domestic households.

\section{REFERENCES}

[1] Danish Ministry of Climate, Energy and Building. "Energy policy report 2012", Tech. Rep. May 2012.

[2] Tanaka, Nobuo. "Technology roadmap: Smart Grids." International Energy Agency, Tech. Rep. 2011.

[3] F. Sossan and H. Bindner, "Evaluation of the Performance of Indirect Control of many DSRs Using Hardware-in-the-loop Simulations". In Decision and Control (CDC2012), IEEE 51st Annual Conference on (pp. 5586-5591). 2012.

[4] Maria van der Hoeven, "Nordic Energy Technology Perspectives: Pathways to a Carbon Neutral Energy Future" 2013 Nordic Energy Technology Perspectives OECD/IEA. Tech. Rep. 2013.

[5] G.T. Costanzo, F. Sossan, M. Marinelli, P. Bacher and H. Madsen, "Grey-box Modeling for System Identification of Household Refrigerators: a Step Toward Smart Appliances", IYCE 2013, Sofiok, Jun 2013. 